\title{
Caractérisation de la variabilité climatique des terroirs viticoles en val de Loire. Influence du paysage et des facteurs physiques du milieu
}

\author{
A Jacquet $^{1 *}, \mathrm{R}_{\text {Morlat }}{ }^{2}$ \\ 'Laboratoire associé Inra Physiologie et biochimie végétales, IRBA, université de Caen, BP 5186, 14032 Caen cedex, France \\ ${ }^{2}$ Inra, URVV, 14070 Beaucouzé, France
}

(Reçu le ?; accepté le 7 janvier 1998)

\begin{abstract}
Summary - Characterization of the climatic variability in the Loire Valley vineyard. Influence of landscape and physical characteristics of the environment. In order to determine the data necessary to establish a climatic mapping of vineyards at the same scale as for soil $(1 / 25000)$, the landscape and climatic environment of 14 vine plots in the Loire Valley have been studied. In addition to topographical characteristics, a synthetic index has been developed: the landscape closing up index (IFP). In a given site, it represents the portion of the celestial canopy shaded by vegetative, topographic or building shields. In the fairly flat relief of this vineyard region, landscape variability is mainly due to vertical vegetative shields and the wide range of soil texture characteristics. This work showed that local climates were associated with geopedology and underlined the effect of the IFP on wind speed (variation greater than 100\%) and on solar radiation interception (variation equal to $10 \%$ ). The recorded temperature differences vary from 0.6 to $3{ }^{\circ} \mathrm{C}$ for vegetative cycle and day time scales, respectively. We observed that they are mainly affected negatively by altitude and positively by sand content in the soil top layer. Air moisture varies according to soil water holding capacity and proximity of forests.
\end{abstract}

vineyard / climate / local climate / mapping / landscape / climatic variability

Résumé - Dans le but de dégager les éléments nécessaires à un zonage climatique des terroirs viticoles à la même échelle cartographique que celui du sol, une caractérisation de l'environnement paysager et du climat de quatorze parcelles représentatives de terroirs du val de Loire a permis d'étudier la formation des mésoclimats rencontrés. Outre les aspects topographiques, un indice synthétique est utilisé : l'indice de fermeture du paysage (IFP). Il représente en un lieu, la portion de la voûte céleste obstruée par des écrans végétaux, topographiques ou bâtis. Dans ce contexte de relief au modelé doux, la variabilité paysagère est essentiellement due aux écrans végétaux verticaux et la gamme de texture de surface des sols est large. Ce travail a permis de mettre en évidence l'existence de mésoclimats associés aux éléments géopédologiques du terroir, et de démontrer l'importance de l'effet de la fermeture du paysage sur la vitesse du vent (variation supérieure à $100 \%$ ) et l'interception du rayonnement solaire (variation de l'ordre de $10 \%$ ). Les différences observées pour la température $\left(0,6^{\circ} \mathrm{C}\right.$ pour l'ensemble du cycle de mesure à $3{ }^{\circ} \mathrm{C}$ pour certaines journées) apparaissent principalement affectées de façon négative par l'altitude et positive par les textures sableuses de la surface des sols. La variabilité de l'humidité de l'air rencontrée correspond aux différences de réserves en eau des sols ou à la présence de forêts proches.

terroir viticole / climat / zonage / paysage / mésoclimat / variabilité climatique 


\section{INTRODUCTION}

L'étude des terroirs viticoles nécessite la mise au point d'une méthode de caractérisation intégrée des facteurs naturels, représentative des conditions de fonctionnement de la vigne. Dans cette optique, le concept d'unité terroir de base (UTB) a été développé (Riou et al, 1995). L'UTB représente un territoire viticole d'extension géographique variable, défini comme l'association des composantes géologique, pédologique et paysagère, et dans lequel la réponse de la vigne est considérée comme reproductible, pour un climat annuel donné (Riou et al, 1995 ; Morlat, 1996).

Dans ce contexte, le milieu édaphique (nature du sol et de la roche) joue un grand rôle sur la morphologie du système racinaire (Morlat et Jacquet, 1993) et les différentes fonctions de ce dernier (extraction de l'eau, absorption minérale, synthèse, mise en réserve, remobilisation de divers composés), avec bien sûr des conséquences importantes sur la physiologie de la vigne (Morlat, 1989)

Mais, compte tenu des exigences de la vigne formulées en termes de température, d'ensoleillement et de bilan hydrique, le climat est aussi une composante majeure du terroir, devant être traitée au même titre que le milieu édaphique. En premier lieu, le profil climatique de l'année de production (millésime) influe sur la composition de la vendange et par conséquent sur les caractéristiques sensorielles du vin. Cette variabilité interannuelle a été étudiée entre autres par Ribéreau Gayon et al (1975), Huglin (1986), Bonnardot (1997).

En viticulture, le zonage climatique a été abordé par de nombreux auteurs. À petite échelle (pays, continent), le macroclimat explique généralement bien la répartition des cépages, la nécessité de certaines pratiques oenologiques (chaptalisation) et les types de vins. À ce niveau, de nombreux auteurs ont mis au point et tenté de valider des indices climatiques représentatifs des potentialités viticoles de telle ou telle région (Winkler, 1962 ; Constantinescu, 1967 ; Huglin, 1978). Riou (1994) a montré, à l'échelle européenne, l'influence positive pour différents cépages, des sommes de températures actives (supérieure à $10^{\circ} \mathrm{C}$ ) calculées pour certaines périodes déterminées du cycle végétatif (en particulier véraison-récolte) sur les teneurs en sucres de la vendange. Dans le cadre d'un essai international d'écologie viticole, Calo et al (1995) relient la teneur en sucres de la vendange, dans le cas des vignobles nord atlantiques, à la température moyenne pendant tout le cycle végétatif, et particulièrement pendant la phase véraison-récolte, de même qu'à l'insolation de cette même période. Les résultats montrent qu'une accumulation significative de sucres se produit lorsque la température moyenne de la phase véraison-récolte augmente jusqu'à un seuil de $17{ }^{\circ} \mathrm{C}$. Entre 17 et $22{ }^{\circ} \mathrm{C}$ la relation n'est plus aussi nette mais reste positive, et au-delà de $22{ }^{\circ} \mathrm{C}$ une tendance négative s'observe.

À l'échelle de l'UTB (quelques hectares à quelques dizaines d'hectares), le zonage précédent n'est plus pertinent, et se pose alors le problème de la caractérisation du climat local associé à l'UTB. Plusieurs composantes de l'UTB sont en mesure de jouer un rôle sur le mésoclimat. Dans la plupart des cas, l'organisation du milieu naturel, permet de situer chaque UTB par rapport à un environnement que l'on peut caractériser par une gamme de variation des altitudes, des pentes, de l'exposition, ainsi que par la nature de la surface du sol, la végétation naturelle...

Les températures décroissent en fonction de l'altitude avec un gradient adiabatique moyen de $-0,65{ }^{\circ} \mathrm{C}$ pour $100 \mathrm{~m}$, en régions tempérées (Guyot, 1997). Par ailleurs, le relief joue un rôle important en influant sur l'écoulement gravitaire de l'air froid le long des pentes ce qui conduit à un abaissement des minima dans les parties basses pouvant atteindre jusqu'à $15{ }^{\circ} \mathrm{C}$ pour $75 \mathrm{~m}$ de dénivellation pour des pentes fortes. La formation d'une ceinture chaude au niveau du tiers supérieur des versants en est la conséquence (Godard, 1949 ; Guyot, 1997).

L'inclinaison des pentes et l'orientation des versants, modifient l'interception du rayonnement solaire (Godard, 1949 ; Varlet Grancher, 1975). Les modifications concernent principalement la fraction directe du rayonnement solaire global pour des pentes inférieures à $30 \%$, et également la fraction diffuse au delà. Une des conséquences est la variation importante de température à la surface du sol (Dumas et al, 1997). Notons que les différences maximales s'observent entre les pentes d'orientation nord et sud et surtout pendant l'hiver. Des modèles de calcul de cette interception ont été mis au point (Durand et Legros, 1981).

Par ailleurs, les pentes affectent également la vitesse du vent. Guyot et al (1976) indiquent qu'une pente de $10 \%$ réduit la vitesse du vent d'environ $10 \%$ au bout de $150 \mathrm{~m}$ lorsqu'il la descend, et l'accélère de $30 \%$ à la même distance lorsqu'il la remonte.

En l'absence de ventilation importante, la température de l'air des basses couches de l'atmosphère dépend des caractéristiques du sol : son albédo ainsi que sa texture et son humidité qui 
affectent sa conductivité et sa capacité thermiques. Ces facteurs modifient le bilan des échanges énergétiques au niveau de la surface (Guyot, 1997).

La végétation naturelle peut influer sur plusieurs variables climatiques (Guyot, 1997) mais dans le cas qui nous intéresse c'est surtout le rôle de brise-vent joué par les haies et les zones boisées. Cet effet a été largement étudié (Guyot, 1963 ; Guyot et al, 1976, 1987). On retiendra que derrière un brise-vent, outre une diminution de la vitesse du vent sur environ 10 fois sa hauteur, on assiste à une augmentation de l'amplitude thermique : augmentation des maximums (et moyennes) et abaissement des minimums. De plus, on observe une réduction du pouvoir évaporant de l'air de 10 à $30 \%$ et des modifications assez complexes des échanges radiatifs en fonction de l'orientation et de l'espacement des haies (Chiapale, 1975 ; Guyot et al, 1987).

Enfin, l'effet des masses forestières sur l'augmentation des précipitations est souvent mentionné (Doignon, 1951 ; Lee, 1978) mais n'a jamais été formellement prouvé.

Ces informations bibliographiques, indispensables à la compréhension des phénomènes climatiques locaux, restent toutefois difficiles à utiliser dans le cadre d'une étude intégrée des terroirs. En effet, à l'échelle de l'UTB les facteurs de variation du climat ont une action conjointe et de ce fait il est difficile de connaître le poids réel de chacun d'eux. Par ailleurs, un zonage climatique direct à cette échelle est inenvisageable. Face à ces problèmes, notre objectif est de proposer puis valider des indicateurs paysagers synthétiques, facilement cartographiables et représentatifs du climat local de l'UTB. Dans cette note, nous abordons les relations entre facteurs physiques de l'environnement paysager et variables climatiques. Cette analyse devrait permettre de déceler les variables utilisables pour une cartographie climatique des terroirs, applicable aux zones tempérées de faible altitude. Il deviendrait ainsi possible d'inclure à la même échelle, et en lui donnant une représentation spatiale, la composante climatique du terroir, au même titre que la composante édaphique (sol, roche). Il serait en outre envisageable, à partir de quelques stations météorologiques de référence, de prédire le climat de parcelles non équipées.

\section{MATÉRIELS ET MÉTHODES}

\section{Le climat régional}

La région, soumise à un climat océanique à tendance septentrionale, se caractérise par une assez faible pluviométrie $(600 \mathrm{~mm})$, des températures moyennes annuelles de l'ordre de $11,5^{\circ} \mathrm{C}$ et des vents dominants ouest et sud-ouest. La durée d'insolation est proche de la limite minimale pour obtenir un vin de qualité, fixée à $1250 \mathrm{~h}$ entre avril et septembre par Ribéreau-Gayon et Peynaud (1971). Les différents indices bioclimatologiques viticoles : degrés-jour de Winkler (1962), produit héliothermique de Branas et al (1946) et l'indice héliothermique de Huglin (1978), classent cette zone dans les vignobles septentrionaux.

\section{Le dispositif expérimental}

Les parcelles expérimentales utilisées ici (14) appartiennent à un réseau de suivi de la vigne, installé en 1979, intégrant la variabilité géologique, pédologique et paysagère des vignobles de Saumur-Champigny, Bourgueil et Chinon situés en moyenne vallée de la Loire (tableau $I$ ).

Tableau I. Caractéristiques géologiques et géomorphologiques de l'ensemble des sites étudiés.

\begin{tabular}{|c|c|c|}
\hline Parcelles & Géologie & Relief \\
\hline IFON & graviers fluviatiles & plaine alluviale - basse terrasse \\
\hline IGAR & colluvions sénoniennes & bas de plateau \\
\hline IBOIS & craie tuffeau - Turonien moyen & bas de plateau \\
\hline $1 \mathrm{ING}$ & sable du Sénonien & versant de plateau \\
\hline 2ING & colluvions sénoniennes & bas de versant de vallée \\
\hline 1CHA & grès et poudingues Eocène continental & sommet de butte \\
\hline 3EL & colluvions sénoniennes & versant sud de plateau \\
\hline $4 \mathrm{EL}$ & argiles du Sénonien & plateau \\
\hline IDAM & craie tuffeau - Turonien moyen & bas de plateau \\
\hline 1POY & craie tuffeau - Turonien moyen & partie inférieure de pente faible \\
\hline 1TUR & limons éoliens & haut de plateau \\
\hline 1BEAU & craie tuffeau - Turonien moyen & plateau dominant \\
\hline 1VAU & limons à silex du Sénonien & haut de plateau \\
\hline 1ROC & argiles caillouteuses du tertiaire & haut de plateau \\
\hline
\end{tabular}




\section{La période de mesure}

Sept années réparties entre 1980 et 1990 ont été étudiées avec ce réseau, mais nous présenterons seulement les résultats de l'année 1988, choisie pour ses valeurs climatiques proches des normales (tableau II). La période de mesure couvre l'ensemble du cycle végétatif de la vigne. Elle s'étale du 22 février au 10 octobre pour la température, l'humidité relative et la pluie et du 18 avril au 10 octobre pour le vent et le rayonnement solaire.

\section{Données météorologiques}

Elles sont collectées dans un poste météorologique standard installé à $2 \mathrm{~m}$ de hauteur au centre de chaque parcelle expérimentale afin qu'il n'occupe pas de position particulière pouvant affecter sa représentativité mésoclimatique (Samie, 1983). Des capteurs de température et d'humidité relative sont placés dans un abri météorologique petit modèle normalisé et reliés à une centrale automatique type Enerco 404 (Cimel, Paris), un anémomètre est aussi connecté à cette centrale. Ce dispositif est complété par un pyranomètre CE180 (Cimel) installé parallèlement à la pente pour la mesure du rayonnement solaire incident (entre 0,3 et $3,0 \mu \mathrm{m}$ ) et par un pluviomètre à lecture directe. Le vent, la température et l'humidité relative sont mesurés à l'échelle horaire et les autres paramètres à l'échelle hebdomadaire. Les différents capteurs ont été régulièrement vérifiés et étalonnés. La précision des capteurs est $\pm 0,2{ }^{\circ} \mathrm{C}$ pour la température et $\pm 3 \%$ pour l'humidité relative et le rayonnement global. La température du point de rosée qui servira à comparer l'humidité de l'air des différents sites est calculée à partir de l'humidité relative et de la température moyenne journalière.

\section{Caractérisation de l'environnement paysager de l'UTB}

Les variables retenues sont celles qui sont susceptibles d'expliquer les différences climatiques mesurées. La pente, l'exposition, l'altitude réelle sont relevées pour chaque site. Le phénomène d'écoulement gravitaire d'air froid déjà évoqué nous a conduits à introduire la notion d'altitude relative qui traduit la position dominante ou dominée d'un site dans son contexte proche. Pour cela, on définit deux paramètres : l'altitude relative globale (ARG) et l'altitude relative selon la pente maximale (ARM). La première est la différence entre l'altitude du site et la moyenne des altitudes relevées sur des cartes IGN au $1 / 25000^{\mathrm{e}}$, tous les $45^{\circ}$ de l'azimut pour des cercles concentriques distants de 100 , $200,300,400$ et $500 \mathrm{~m}$ du centre de la parcelle (soit 40 points). La seconde procède du même calcul, mais seulement pour la direction correspondant à la pente maximale de la zone (soit 10 points), les valeurs sont logiquement positives pour une butte ou un plateau, négatives pour une vallée. Ces calculs peuvent s'automati-
Tableau II. Comparaison du climat de l'année 1988 aux normales (1962-1990).

\begin{tabular}{lcc}
\hline & Normales & 1988 \\
\hline Température & $11,5^{\circ} \mathrm{C}$ & $12,3{ }^{\circ} \mathrm{C}$ \\
Humidité relative & $81,2 \%$ & $83,1 \%$ \\
Précipitation & $620 \mathrm{~mm}$ & $704 \mathrm{~mm}$ \\
Vitesse moyenne du vent & $3,6 \mathrm{~m} / \mathrm{s}$ & $3,3 \mathrm{~m} / \mathrm{s}$ \\
Insolation & $1944 \mathrm{~h}$ & $1827 \mathrm{~h}$ \\
\hline
\end{tabular}

Tableau III. Classes de fermeture du paysage en fonction d'IFP (angles solides exprimés en stéradians).

\begin{tabular}{lc}
\hline IFP $360^{\circ}$ (en sr) & Classes de fermeture \\
\hline $0,00-0,25$ & ouvert \\
$0,25-0,50$ & assez ouvert \\
$0,50-1,00$ & assez fermé \\
$>$ à 1,00 & fermé \\
\hline
\end{tabular}

ser à partir d'un système d'information géographique ( $\mathrm{Sig}$ ) et d'un modèle numérique de terrain.

Pour quantifier les effets brise-vent et de masque de l'environnement paysager, un indice de fermeture du paysage (IFP) est utilisé. Cet indice a été introduit par Morlat (1989), puis développé et précisé par Jacquet et al (1995), et Jacquet et Morlat (1996). Il est déterminé à partir de mesures d'angles réalisées au théodolite tous les cinq grades d'azimut. Les valeurs obtenues donnent par intégration un IFP exprimé en angles solides pour $360^{\circ}$ (tableau III), mais aussi pour $180^{\circ}$ nord (IFPN) et $180^{\circ}$ sud (IFPS) et dont les valeurs varient entre 0 et 2 stéradians (l'hémisphère céleste correspondant à 2(sr). Cet indice représente donc en un lieu, la portion de la voûte céleste occultée par des écrans végétaux, la topographie ou des bâtiments. Cette méthode de calcul est plus précise que celle présentée par Lebon (1993) qui n'utilise que huit mesures de hauteur angulaire au clysimètre par point d'observation, mais n'est pas aussi facile à mettre en æuvre et donc moins bien adaptée à la cartographie des terroirs.

Pour connaître la fraction du rayonnement global réfléchie par le sol, des mesures d'albédo du sol ont été réalisées par ciel clair et sol sec au moyen de deux pyranomètres CM6B (Kipp et Zonen, Delft, Pays-Bas). La texture et la charge en cailloux de l'horizon de surface ont été déterminées par analyse mécanique et granulométrique, dans le but d'étudier leur influence sur la température de l'air. La consommation en eau du système sol-plante qui peut affecter la teneur en eau de l'air a été mesurée à l'aide d'un humidimètre neutronique (type Solo 25S) sur la durée du cycle végétatif.

\section{Traitement des données}

Pour dégager les facteurs locaux du climat les plus discriminants, une analyse en composantes principales 
(ACP) est réalisée sur les variables physiques des terroirs préalablement décrites.

Les données climatiques sont traitées à différentes échelles temporelles : depuis le profil journalier horaire jusqu'à l'année (cycle végétatif) ainsi que par type de temps. À l'exception des précipitations, nous avons choisi d'exprimer les résultats concernant les variables climatiques de chaque site, sous forme d'écarts à la moyenne calculée sur l'ensemble des sites. Nous appellerons cette moyenne « référence régionale ».

En prenant comme base les travaux de Lebon (1993), nous avons caractérisé les types de temps par 24 combinaisons entre : trois classes d'insolations caractérisées par le quotient $\mathrm{Q}^{\mathrm{g}}$ du rayonnement global mesuré par le rayonnement global théorique aux confins de l'atmosphère $\left(\mathrm{Q}^{\mathrm{g}} \leq 0,3\right.$ : temps couvert ; $0,3<\mathrm{Q}^{\mathrm{g}} \leq 0,6$ : temps variable ; $\mathrm{Q}^{\mathrm{g}}>0,6$ : temps ensoleillé), deux classes de vitesse du vent (temps calme : vent $<2 \mathrm{~m} \mathrm{~s}^{-1}$; venté au delà, et quatre classes de direction du vent (NO à NE; NE à SE; SE à SO ; SO à NO).

Les occurrences de chaque combinaison sont présentées sur le tableau IV. En 1988, les journées calmes sont majoritaires $(56,4 \%)$ et les flux les plus représentés sont respectivement ouest $(47,9 \%)$ et sud $(22,6 \%)$.
Par ailleurs, les journées variables dominent largement la période $(47,8 \%)$, suivies par le temps ensoleillé surtout représenté par temps calme avec $31,6 \%$.

\section{RÉSULTATS}

\section{Caractérisation des UTB par leurs principaux facteurs physiques}

La région a un modelé doux avec de faibles amplitudes topographiques (altitudes comprises entre 42 et $108 \mathrm{~m}$ ), les pentes sont faibles à nulles (< à $5 \%$ ) ; les valeurs d'altitude relative (inférieures à $13 \mathrm{~m}$ ) confirment cette observation (tableau $\mathrm{V}$ ). La plupart des sites sont ouverts vers le sud et protégés au nord.

Le plan principal 1, 2 fourni par l'ACP (fig l) dont la matrice est contenu dans le tableau $V$, explique $66,7 \%$ de la variation totale des facteurs physiques du terroir, retenus pour l'étude. Sur

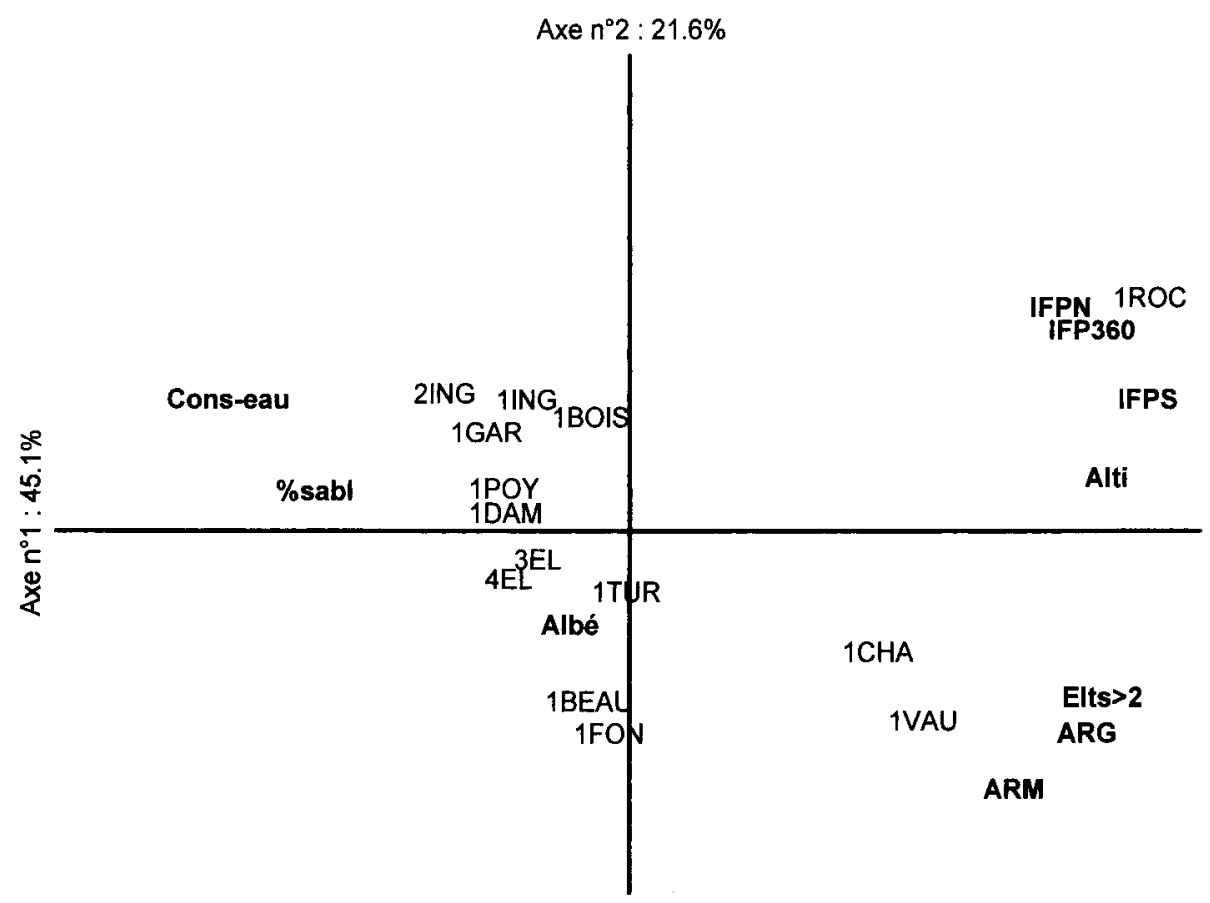

Fig 1. Analyse en composantes principales des facteurs physiques de variation du mésoclimat dans les vignobles de la moyenne vallée de la Loire réalisée à partir de quatorze sites représentatifs des terroirs présents.

Tableau IV. Types de temps observés en 1988, poste météorologique de Montreuil-Bellay (en \% jours).

\begin{tabular}{|c|c|c|c|c|c|c|c|c|c|}
\hline \multirow[b]{2}{*}{ Fréquence } & \multicolumn{4}{|c|}{ Journées calmes } & \multicolumn{4}{|c|}{ Journées ventées } & \multirow[b]{2}{*}{ Total } \\
\hline & Variable & Couvert & Ensoleillé & Sous-tot & Variable & Couvert & Ensoleillé & Sous-tot & \\
\hline Vent Nord & 2,6 & 1,3 & 1,7 & 5,6 & 3,8 & 0,8 & 1,7 & 6,4 & 12,0 \\
\hline Vent Est & 2,6 & 1,7 & 6,0 & 10,3 & 2,1 & 0,4 & 4,7 & 7,3 & 17,5 \\
\hline Vent Sud & 7,7 & 2,6 & 5,1 & 15,4 & 3,0 & 3,4 & 0,8 & 7,3 & 22,6 \\
\hline Vent Ouest & 12,4 & 3,0 & 9,8 & 25,2 & 13,7 & 7,3 & 1,7 & 22,6 & 47,9 \\
\hline Sous - total & 25,2 & 8,5 & 22,6 & 56,4 & 22,6 & 12,0 & 9,0 & 43,6 & 100 \\
\hline
\end{tabular}


Tableau V. Principales caractéristiques de l'environnement paysager des parcelles étudiées.

\begin{tabular}{|c|c|c|c|c|c|c|c|c|c|c|c|c|c|}
\hline & \multirow[b]{2}{*}{ parcelles } & \multicolumn{5}{|c|}{ Topographie } & \multicolumn{3}{|c|}{$\begin{array}{c}\text { Ouverture / fermeture } \\
\text { du paysage }\end{array}$} & \multicolumn{3}{|c|}{$\begin{array}{c}\text { État de surface } \\
\text { du sol }\end{array}$} & \multirow{2}{*}{$\begin{array}{l}\text { Consommation } \\
\text { en eau en mm } \\
\begin{array}{c}\text { cons en eau } \\
\text { du } 0106 \\
\text { au } 101088\end{array}\end{array}$} \\
\hline & & $\begin{array}{l}\text { altitude } \\
\text { en } m\end{array}$ & $\begin{array}{l}A R G \\
\text { en } m\end{array}$ & $\begin{array}{l}\text { ARM } \\
\text { en } m\end{array}$ & $\begin{array}{l}\text { déclivité } \\
\text { en } \%\end{array}$ & orientation & $\begin{array}{c}I F P N \\
180^{\circ} \mathrm{Nor}\end{array}$ & $\begin{array}{c}\text { IFPS } \\
180^{\circ} \mathrm{Sud}\end{array}$ & $\begin{array}{l}I F P \\
360^{\circ}\end{array}$ & $\begin{aligned} & \% \text { élts } \\
> & \text { à } 2 \mathrm{~mm}\end{aligned}$ & $\begin{array}{c}\% \\
\text { sable }\end{array}$ & albédo & \\
\hline $\mathrm{a}$ & $1 \mathrm{FON}$ & 42 & 6,3 & 5,7 & néant & - & 0,07 & 0,11 & 0,18 & 31 & 88,0 & 24,5 & 264 \\
\hline $\mathrm{a}$ & IGAR & 57 & $-2,7$ & $-5,3$ & néant & - & 0,36 & 0,09 & 0,46 & 5 & 68,3 & 22,0 & 375 \\
\hline b & 1BOIS* & 83 & 4,9 & $-0,8$ & 2 & $\mathrm{~S}$ & 0,36 & 0,17 & 0,52 & 2 & 79,5 & 21,0 & 423 \\
\hline b & IING & 66 & 1,3 & $-3,9$ & 3 & $S$ & 0,47 & 0,08 & 0,55 & 4 & 91,6 & 23,5 & 385 \\
\hline b & $2 \mathrm{ING}^{*}$ & 63 & $-3,3$ & $-3,7$ & néant & - & 0,27 & 0,12 & 0,39 & 0 & 91,0 & 23,5 & 392 \\
\hline c & $1 \mathrm{CHA}$ & 74 & 13,0 & 10,4 & 4,5 & $N-W$ & 0,45 & 0,23 & 0,67 & 25 & 76,4 & 22,0 & 236 \\
\hline $\mathrm{c}$ & $3 \mathrm{EL}$ & 67 & 1,4 & 5,2 & 5 & $\mathrm{~S}$ & 0,17 & 0,11 & 0,29 & 1 & 87,4 & 25,0 & 332 \\
\hline $\mathrm{c}$ & $4 \mathrm{EL}$ & 75 & 4,4 & 4,1 & 2 & $\mathrm{~N}$ & 0,10 & 0,12 & 0,23 & 2 & 76,6 & 26,0 & 386 \\
\hline c & 1DAM & 52 & 0,9 & 0,3 & néant & - & 0,10 & 0,04 & 0,14 & 7 & 62,6 & 21,0 & 376 \\
\hline$c$ & IPOY & 46 & $-0,3$ & $-3,4$ & 3 & $\mathrm{~S}$ & 0,26 & 0,09 & 0,35 & 5 & 71,7 & 21,0 & 312 \\
\hline$c$ & ITUR & 83 & 4,6 & 0,1 & néant & - & 0,04 & 0,08 & 0,12 & 11 & 39,4 & 20,7 & 339 \\
\hline $\mathrm{d}$ & 1BEAU & 58 & 10,5 & 10,4 & 2 & $S$ & 0,02 & 0,04 & 0,07 & 6 & 70,0 & 21,0 & 370 \\
\hline d & 1VAU & 103 & 8,9 & 5,3 & néant & - & 0,27 & 0,18 & 0,45 & 63 & 37,8 & 23,6 & 257 \\
\hline $\mathrm{d}$ & $1 \mathrm{ROC}^{*}$ & 108 & 8,4 & 6,6 & néant & - & 1,04 & 0,74 & 1,78 & 22 & 50,2 & 22,0 & 274 \\
\hline
\end{tabular}

$\mathrm{a}=$ Saint-Nicolas-de-Bourgueuil, $\mathrm{b}=$ Bourgueuil, $\mathrm{c}=$ Saumur-Champigny, $\mathrm{d}=$ Chinon $; \mathrm{a}$ et $\mathrm{b}=$ Nord Loire, $\mathrm{c}$ et $\mathrm{d}=$ Sud Loire . $\mathrm{ARG}=$ altitude relative globale, $\mathrm{ARM}=$ altitude relative selon la pente maximum. $\mathrm{IFP}=$ indice de fermeture du paysage en stéradians (sr). * présence d'une forêt importante à proximité.

l'axe 1 qui apparaît le plus discriminant, les variables les plus contributives sont les différents indices de fermeture du paysage, l'altitude vraie et relative ainsi que la teneur en cailloux des sols ; celles-ci s'opposent à la teneur en sable des sols et à la consommation en eau du système terroir-vigne. La position stratigraphique, la nature et la dureté des matériaux géologiques de certains terroirs (grès et poudingues pour 1CHA, argile très caillouteuse à blocs rocheux pour 1ROC, limons à silex pour 1VAU), expliquent bien les relations entre fortes altitudes réelles et relatives, pierrosité importante et indice de fermeture du paysage élevé. En effet, ces terroirs difficiles à cultiver de part leur nature se rencontrent souvent à proximité de zones boisées.

La composante végétale de l'IFP domine largement et détermine majoritairement la fermeture du paysage. Cette végétation est un trait caractéristique de certains terroirs viticoles et sa présence est souvent liée à la nature physicochimique du substrat (Morlat, 1989). L'albédo des sol (de 20,7 à $26 \%$ ) ne permet pas de bien discriminer les parcelles. En revanche, le pourcentage de sables du premier horizon présente une grande variabilité (de 37,8 à 91,6\%).

En croisant les résultats de l'ACP et les informations du tableau $V$, on peut distinguer plusieurs groupes de situations caractéristiques : IFON,

- à paysage très ouvert : 1BEAU, ITUR,

- à paysage fermé : 1ROC, 1CHA,

- dont l'horizon de surface est riche en cailloux : IVAU, 1CHA, IFON,

- proches d'un environnement forestier important : 1ROC, 1BOIS, 2ING.

\section{Variabilité du climat des UTB}

\section{La pluviométrie}

La comparaison entre sites doit être prudente car une fraction des précipitations est tombée sous forme d'averses orageuses dont la distribution reste aléatoire. Toutefois, la tendance observée sur 20 ans entre les postes météo Nord-Loire (Bourgueil : $636 \mathrm{~mm} / \mathrm{an}$ ) et Sud Loire (Saumur : $584 \mathrm{~mm} / \mathrm{an}$, Chinon : $615 \mathrm{~mm} / \mathrm{an}$ ) est confirmée. En effet, les parcelles de Saumur et Chinon (groupe $c$ et d du tableau $V$ ) ont reçu le moins de précipitations (total entre 310 et $330 \mathrm{~mm}$ ), alors que celles de Bourgueil, groupe b, se situent entre 395 et $434 \mathrm{~mm}$ sur la période d'étude. Seules 1FON (Nord Loire) et 1TUR (Sud Loire) occupent une position intermédiaire. Les valeurs du suivi hebdomadaire n'apportent pas d'information supplémentaire notable. 


\section{Le rayonnement solaire incident}

À l'échelle de la période d'étude, les écarts maximaux de rayonnement global incident $(\mathrm{Rg})$ reçu entre parcelles atteignent $280 \mathrm{MJ} / \mathrm{m}^{2}$ pour une exposition énergétique globale moyenne totale de $2856 \mathrm{MJ} / \mathrm{m}^{2}$, soit l'équivalent de $16 \mathrm{j}$ d'éclairement moyen (fig 2). La plus ensoleillée, ITUR, s'oppose à $1 \mathrm{ROC}$ et $1 \mathrm{CHA}$. Les écarts hebdomadaires à la référence atteignent respectivement $+6 \%$ et $-8 \%$ pour les semaines anticycloniques de l'été. Certaines parcelles, comme 1FON, 3EL, 2ING, présentent des variations autour de la référence. Par temps très clair (fort rayonnement global) 2ING rejoint le groupe des sites les plus éclairés.

\section{La vitesse du vent}

C'est le paramètre le plus affecté dans ce réseau expérimental. Lebon (1993), en Alsace, a trouvé des résultats similaires. Les parcelles 1 ROC et
1CHA sont les moins ventées ; à l'opposé, 1TUR et 1BEAU présentent les vitesses maximales (fig 3). Entre ces extrêmes, on observe une augmentation de vitesse du vent sur 1ING, pour les flux de secteur $\mathrm{S}-\mathrm{O}$ à $\mathrm{O}$ et une diminution sur 1 BOIS pour les flux N à S-E (fig 4). Pour des journées type de fort flux $\mathrm{O}$ à $\mathrm{S}-\mathrm{O}$ (30 mai et 6 octobre), des écarts à la référence de $+20 \%$ pour 1BEAU, 1TUR, 1ING et de $-60 \%$ pour 1 ROC ont été enregistrés. Pour des flux de N-E (6 mai), $1 \mathrm{BEAU}$ et 2ING présentent les plus fortes augmentation (+40\% par rapport à la référence) tandis que l'on note des réductions de $70 \%$ sur 1 ROC et $35 \%$ pour 1 BOIS.

\section{L'humidité de l'air}

Pour l'analyse de la variabilité de l'humidité de l'air, on utilisera la température du point de rosée qui représente la température à laquelle il faut refroidir l'air pour que la tension de vapeur d'eau devienne saturante (les valeurs les plus élevées
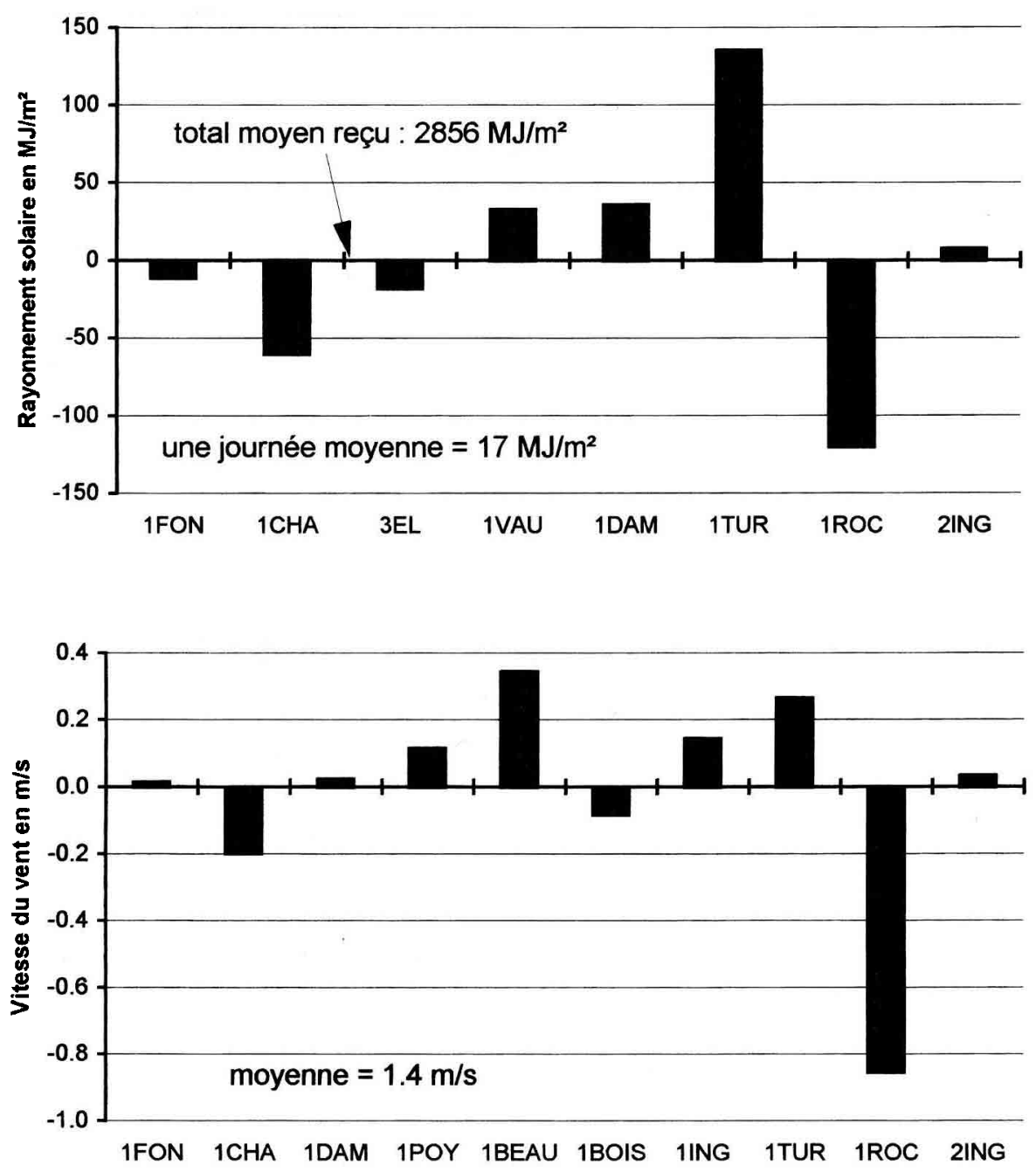

Fig 2. Variabilité du rayonnement solaire incident intercepté pour huit sites du réseau terroir entre le 26 avril 1988 et le 10 octobre 1988.
Fig 3. Vitesse moyenne du vent en écart à la référence du 26 avril 1988 et le 10 octobre 1988. 

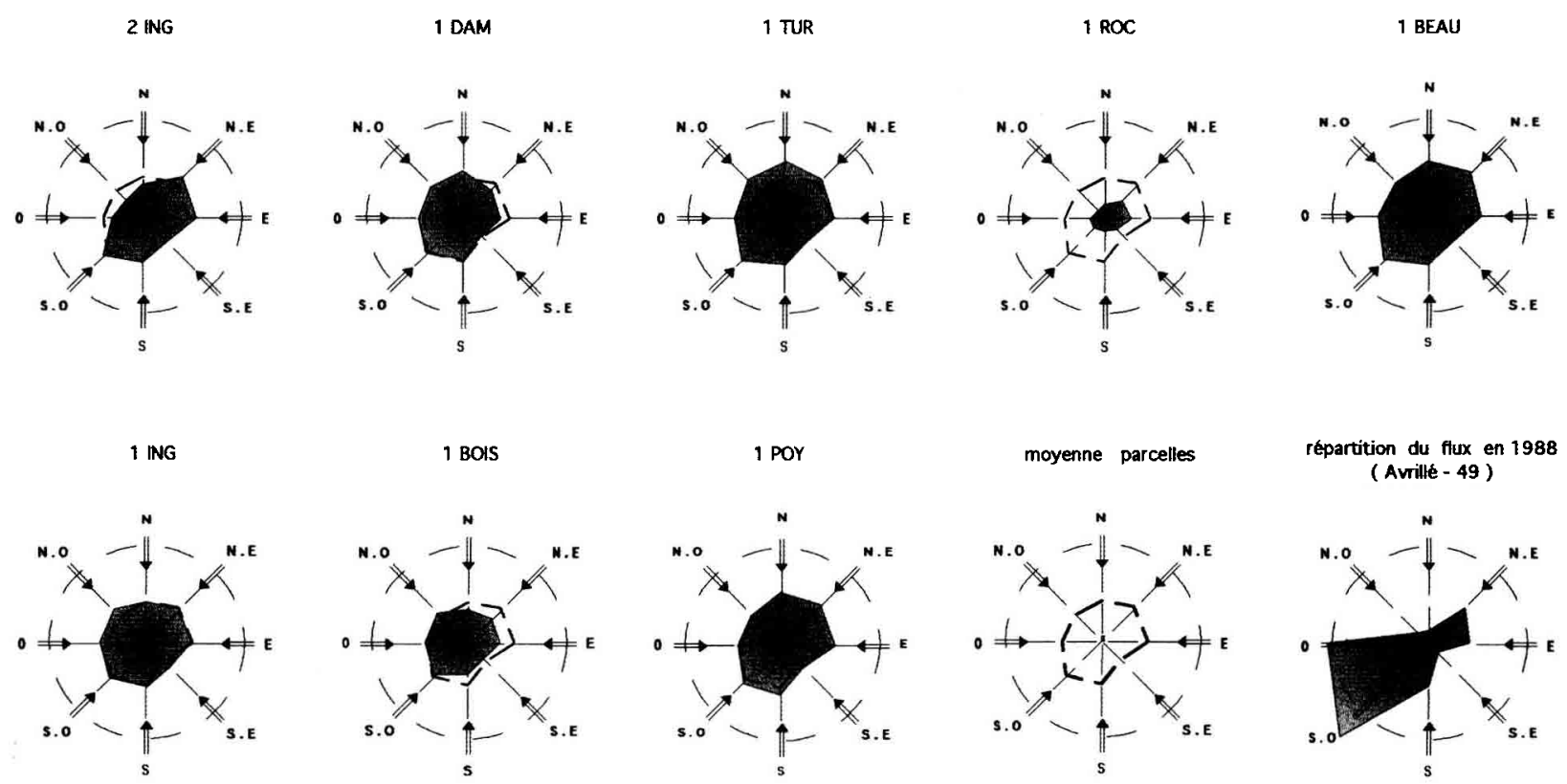

Fig 4. Vitesse moyenne du vent selon les secteurs pour huit sites du réseau. Les longueurs portées sur chacun des axes sont proportionnelles aux vitesses mesurées. La courbe en tirets correspond à la moyenne des huit sites. Le rayon du cercle correspond à une vitesse de $3,2 \mathrm{~m} / \mathrm{s}$.

correspondent aux teneurs en eau de l'air les plus fortes). Les durées pendant lesquelles l'humidité relative est inférieure à $40 \%$ serviront à comparer les sites par rapport à la fréquence des périodes sèches. Le nombre d'heures pendant lequel l'humidité relative est supérieure à $80 \%$ permettra de mettre en évidence les zones où le risque de développement de maladies cryptogamiques est plus grand.

À l'échelle du cycle végétatif, la température moyenne du point de rosée varie de 10,1 à $11,6^{\circ} \mathrm{C}$ selon les terroirs. Parmi les parcelles les plus humides on trouve 1ROC, 2ING suivies de 1BOIS qui affichent respectivement un point de rosée supérieur à la référence pour 99,73 et $71 \%$ des jours (tableau VI). Les plus faibles températures du point de rosée se rencontrent sur 1CHA, IVAU et 1 POY, ces sites comptent respectivement 82,78 et $67 \%$ de jours avec une température du point de rosée inférieure à la référence, 4EL et 1TUR sont aussi plus « secs » (tableau VI).

Les suivis hebdomadaires des écarts à la moyenne régionale (fig 5) ont permis de distinguer un groupe de parcelles où la température du point de rosée croît avec le temps, composé de 1DAM, $1 \mathrm{POY}, 1 \mathrm{BOIS}, 1 \mathrm{BEAU}$ opposé à un groupe où elle décroît au cours du temps (1CHA, IVAU, 1FON). On notera l'homogénéité des résultats obtenus dans les parcelles sur craie tuffeau (tableau I) pour ce paramètre.

Les différences de température de rosée se manifestent surtout à partir de mai et se produisent essentiellement pendant la phase diurne et au coucher du Soleil. Les différences entre types de temps advectifs au sein d'une même UTB peuvent être aussi importantes que les écarts entre sites extrêmes pour un même type de temps (environ 10 à $15 \%$ d'écart entre la température du point de

Tableau VI. Principales caractéristiques de l'humidité de l'air pour les différents terroirs exprimées en écarts à la référence $^{1}$ et en $\%$ de jours où la valeur est supérieure à cette référence ${ }^{2}$, du 22 février au 10 octobre 1988.

\begin{tabular}{|c|c|c|c|c|c|c|c|c|c|c|c|c|c|c|c|}
\hline & $M O Y$ & $1 F O N$ & $I G A R$ & $I B O I$ & $I I N G$ & $2 I N G$ & ICHA & $3 E L$ & $4 E L$ & $I D A M$ & $I P O Y$ & ITUR & $I B E A$ & IVAU & $J 1 R O C$ \\
\hline Temp pt de roséel & 10,7 & $+0,3$ & $+0,1$ & $+0,3$ & $+0,3$ & $+0,2$ & $-0,5$ & $-0,2$ & $-0,2$ & $+0,2$ & $-0,4$ & $-0,4$ & $+0,2$ & $-0,6$ & $+0,8$ \\
\hline \multicolumn{16}{|c|}{ Temp pt de rosée } \\
\hline$>$ à la moyenne ${ }^{2}$ & - & 68 & 65 & 71 & 79 & 73 & 19 & 42 & 41 & 79 & 33 & 26 & 72 & 21 & 99 \\
\hline Heures $>80 \%$ & 3274 & -10 & -55 & +220 & +94 & +310 & -55 & -80 & -190 & +60 & -227 & -190 & +65 & -200 & +410 \\
\hline Heures $<40 \% 1$ & 124 & -100 & -55 & -7 & -37 & -33 & +17 & +48 & -9 & -25 & +116 & +80 & +15 & +47 & -59 \\
\hline
\end{tabular}


rosée moyenne du flux calme de nord et celle des forts flux de sud pour 2ING).

Le nombre d'heures où l'humidité relative est inférieure à $40 \%$ fait simplement apparaître les parcelles 1FON comme plus humide (écart négatif de $100 \mathrm{~h}$ par rapport aux $124 \mathrm{~h}$ de la référence) et 1 POY comme plus sèche (écart positif de $116 \mathrm{~h}$ ).

Les risques de développement de maladies cryptogamiques apparaissent plus élevés dans des terroirs comme 1ROC, 2ING, 1BOIS, qui présentent respectivement 410,310 et 220 h d'humidité relative $>$ à $80 \%$ de plus que la référence, que dans ceux comme 1POY, 4EL et 1TUR dont les valeurs lui sont inférieures d'environ $200 \mathrm{~h}$.

\section{La température}

À l'échelle de la période entière de mesure, la gamme de variation entre les parcelles est de $0,7^{\circ} \mathrm{C}$ pour la température moyenne (tableau VII).
Elle correspond à l'écart qui existe entre le site le plus froid (2ING avec $13,7{ }^{\circ} \mathrm{C}$ ) et ceux les plus chauds ( $1 \mathrm{FON}$ et $1 \mathrm{GAR}$ avec $14,4^{\circ} \mathrm{C}$ ). Les autres parcelles ont des températures comprises entre 13,9 et $14,3{ }^{\circ} \mathrm{C}$. Si l'on s'intéresse au pourcentage des jours où la température moyenne des parcelles est supérieure de $0,5{ }^{\circ} \mathrm{C}$ à la moyenne générale (tableau VII), on note une plus forte variation entre sites et on peut discriminer cinq parcelles plus chaudes qui sont : 1FON, 1GAR, 3EL, 1POY, 1ROC.

Les écarts atteignent $1,2^{\circ} \mathrm{C}$ pour les minimums, la parcelle la plus froide est $2 \mathrm{ING}\left(8,7^{\circ} \mathrm{C}\right)$ qui s'oppose à deux parcelles plus chaudes (4EL et 1 ROC avec $9,9^{\circ} \mathrm{C}$ ). Pour les maximums, l'écart atteint $1{ }^{\circ} \mathrm{C}$, avec le site 1 VAU plus froid $\left(18,7^{\circ} \mathrm{C}\right)$ et $1 \mathrm{ROC}$ plus chaud $\left(19,7^{\circ} \mathrm{C}\right)$, la parcelle $2 \mathrm{ING}$ ne s'individualise plus.

Les parcelles qui présentent les plus fortes amplitudes thermiques moyennes $\left(10,3{ }^{\circ} \mathrm{C}\right.$; $10,0{ }^{\circ} \mathrm{C}$ ) sont $2 \mathrm{ING}$ et $1 \mathrm{POY}$, et celles qui mon-
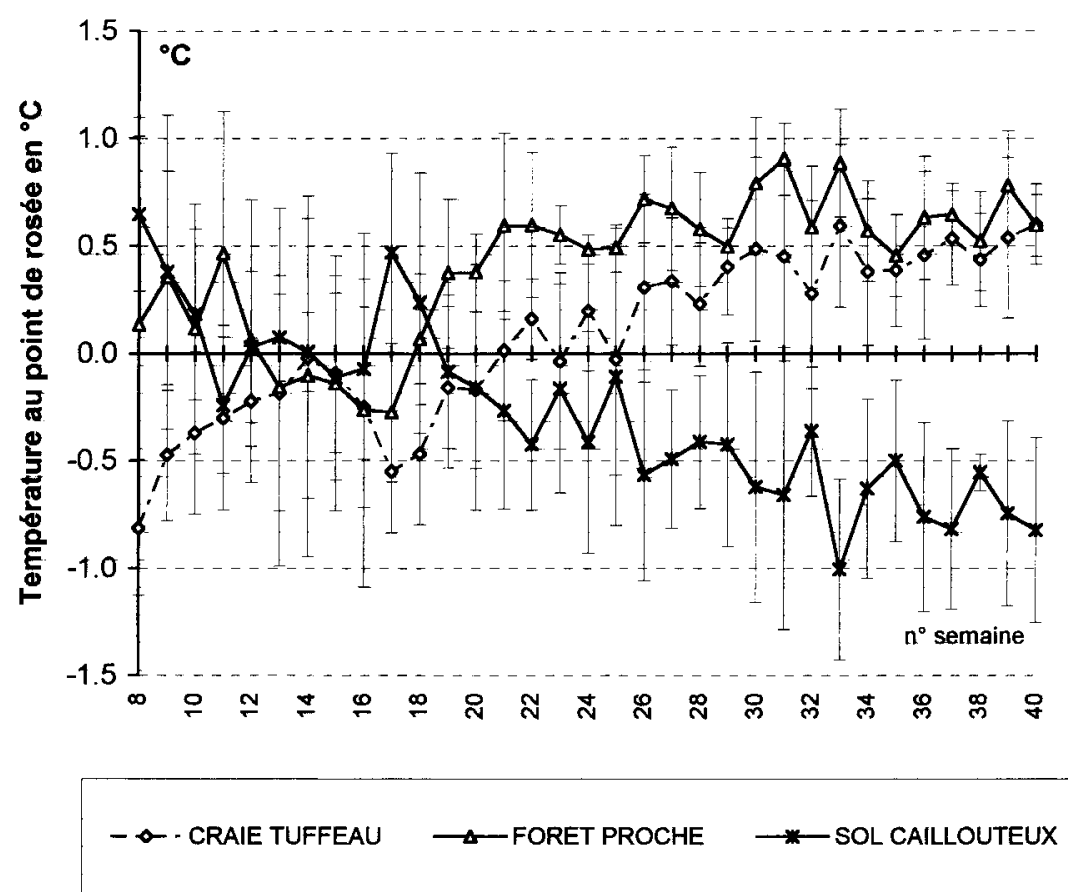

Fig 5. Évolution des écarts hebdomadaires de température moyenne du point de rosée entre trois groupes de parcelles et la référence régionale.

Tableau VII. Principales caractéristiques de la température de l'air pour les différents terroirs exprimées en valeurs moyennes ${ }^{1}$ et en $\%$ de jours où la température moyenne journalière est supérieure de $0,5^{\circ} \mathrm{C}$ à la référence ${ }^{2}$, du 22 février au 10 octobre 1988 .

IFON IGAR IBOIS IING 2ING ICHA 3EL 4EL IDAM IPOY ITUR IBEAUIVAU IROC

\begin{tabular}{|c|c|c|c|c|c|c|c|c|c|c|c|c|c|c|}
\hline Température moyenne ${ }^{1}$ & 14,4 & 14,3 & 14,0 & 14,2 & 13,7 & 14,1 & 14,3 & 14,2 & 14,1 & 14,2 & 14,1 & 14,2 & 13,9 & 14,2 \\
\hline Température minimale ${ }^{1}$ & 9,7 & 9,8 & 9,6 & 9,8 & 8,6 & 9,6 & 9,7 & 9,8 & 9,7 & 9,4 & 9,8 & 9,7 & 9,5 & 9,8 \\
\hline Température maximale & 19,2 & 19,1 & 19,0 & 19,0 & 19,0 & 18,9 & 19,0 & 18,8 & 19,1 & 19,4 & 18,9 & 19,2 & 18,6 & 19,3 \\
\hline Amplitude & 9,5 & 9,3 & 9,4 & 9,2 & 10,3 & 9,3 & 9,4 & 9,0 & 9,4 & 10,0 & 9,1 & 9,5 & 9,0 & 9,5 \\
\hline$\%$ jours Tmoy $>2$ & 20 & 27 & 4 & 14 & 0 & 5 & 20 & 14 & 5 & 20 & 6 & 12 & 6 & 22 \\
\hline
\end{tabular}


trent les plus faibles $\left(9{ }^{\circ} \mathrm{C}\right)$ sont $1 \mathrm{VAU}$ et $4 \mathrm{EL}$ (tableau VII).

Outre une légère accentuation (de 0,1 à $0,2^{\circ} \mathrm{C}$ ) des différences déjà observées, pour les temps calmes et ensoleillés, l'approche par type de temps n'apporte pas de renseignements complémentaires.

À l'échelle horaire, des différences de comportement notables apparaissent. Celles-ci donnent en partie la manière dont les écarts observés à l'échelle du cycle végétatif se forment. La journée du 25 juillet 1988, représentative des situations avec un temps calme et un ciel clair, en fournit une illustration pour cinq parcelles (fig 6). On peut y constater des écarts de plus de $3{ }^{\circ} \mathrm{C}$ entre parcelles pour certaines heures de la journée.

\section{Discussion}

Dans le cadre de notre étude, le sol est une composante du terroir qui varie largement d'un site à l'autre et qui a été abordée en détail par Morlat (1989). On a pu mettre en évidence une relation positive entre la température de l'air et la teneur en sables totaux du premier horizon du sol ( $f i g$ 7), avec un coefficient de corrélation qui avoisine 0,8 si l'on retire la parcelle 2ING située dans un couloir d'air froid. La nature du sol peut jouer un rôle sur le climat en intervenant sur le bilan d'énergie par l'effet de son albédo (mais qui varie peu dans cette étude comme le montre le tableau $V$ ) sur le rayonnement net, et aussi en modifiant le devenir de la quantité de chaleur stockée en raison de sa capacité calorifique et de sa conductivité thermique. Les résultats que nous avons obtenus par ailleurs (Cellier et al, 1996), montrent que le potentiel de réchauffement des sols est lié à sa capacité calorifique volumique et à sa conductivité thermique. La capacité calorifique volumique du sol dépend en grande partie de sa teneur en eau, généralement plus faible pour les textures sableuses. La conductivité thermique est une fonction complexe des caractéristiques texturales et structurales du sol (de Vries, 1963). On peut estimer que les sols sableux en se desséchant plus vite que les sols limoneux ou argileux et en ayant une porosité en général plus forte, auront une conductivité thermique qui sera plus faible. Il en résultera que pendant la journée la température de l'horizon de surface sera plus élevée en sol sableux. À titre d'exemple, la température moyenne à $-5 \mathrm{~cm}$ calculée sur la période de mars à juillet 1988 a été de $15,9{ }^{\circ} \mathrm{C}$ pour des sols sableux (1FON, 3EL) contre seulement $14,2{ }^{\circ} \mathrm{C}$ pour un sol argileux comme 1VAU (Restoin, 1993). On peut donc penser que la plus forte température de surface des sols sableux induit un rayonnement infrarouge thermique plus important qui pourrait expliquer en grande partie la relation positive citée plus haut.

À une autre échelle, les courbes représentant les écarts thermiques horaires à la référence régionale, calculés pour cinq sites représentatifs lors d'une journée représentative (fig 6), mettent en évidence des comportements pouvant être reliés pour partie à l'effet du sol. La courbe a une allure croissante pour le site 1 FON qui de plus frais au lever du soleil devient nettement plus chaud le soir et également pour 1VAU dans une gamme de
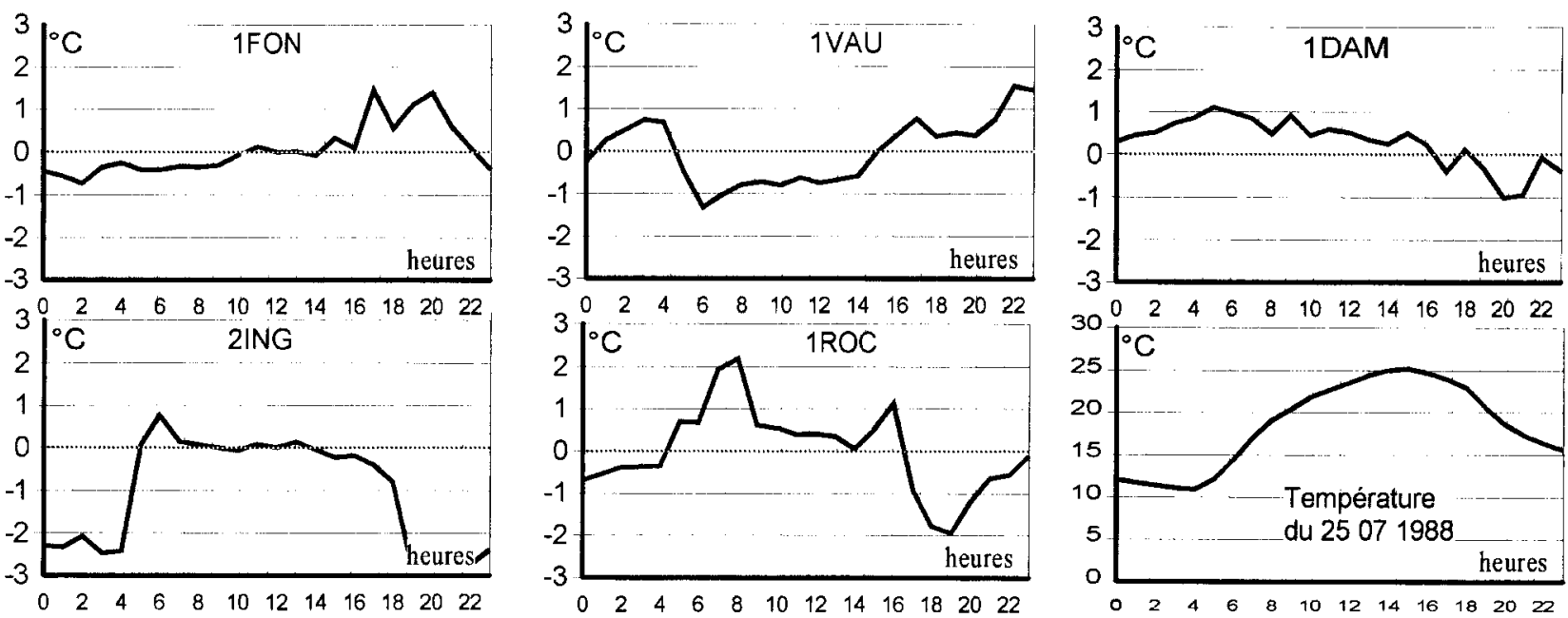

Fig 6. Écarts thermiques horaires à la référence régionale calculés pour cinq sites représentatifs dans le cas d'une journée calme avec ciel clair (25 juillet 1988). 


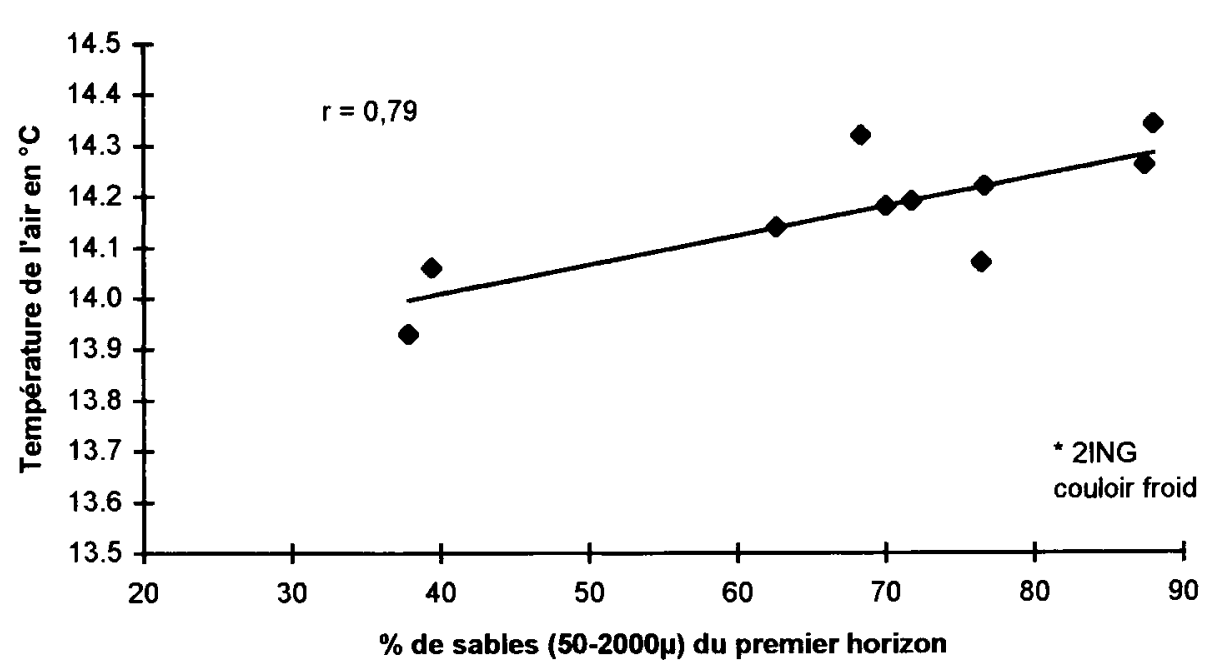

Fig 7. Variation de la température moyenne de l'air en fonction de la teneur en sables de l'horizon de surface pour dix terroirs sur l'ensemble du cycle végétatif de la vigne en 1988.

valeurs de température inférieures. Le comportement de ces parcelles est à rattacher, pour le matin à la mauvaise conductivité thermique de leur sol caillouteux et pour le soir, à la restitution de l'énergie emmagasinée au cours de la journée par les graviers et cailloux de surface de ces sols. On observe l'inverse sur la parcelle IDAM dont le sol, constitué d'éléments fins, est bon conducteur thermique. Ceci rejoint les observations de Verbrugghe et al (1991) qui ont mesuré, dans une même unité parcellaire, des températures plus fortes pour les grappes situées au dessus de sols de galets ou de pierres concassées que pour celles établies au dessus d'un sol constitué de particules fines.

Indirectement, les réserves disponibles en eau du sol en modulant l'évapotranspiration réelle du couvert, peuvent influer sur l'humidité de l'air des basses couches de l'atmosphère, surtout en période peu ventée. On peut remarquer qu'une augmentation au cours de l'année de la température du point de rosée par rapport à la référence, existe pour les sites sur craie tuffeau (IDAM, 1POY, 1BOIS, 1BEAU) (fig 5) dans lesquels la consommation en eau du système sol / vigne sur le cycle végétatif, a été en général bien supérieure à $300 \mathrm{~mm}$ (tableau V). Le comportement opposé s'observe pour les parcelles caillouteuses et sablocaillouteuses (1VAU, 1FON, 1CHA) qui présentent toutes une consommation en eau bien inférieure à $300 \mathrm{~mm}$. Ce phénomène s'exprime aussi sur la durée des périodes sèches (nombre d'heures avec une humidité relative inférieure à $40 \%$ ) entre les parcelles IFON et IPOY

L'indice de fermeture du paysage (IFP), est proposé et calculé afin de tenir compte, notamment, des effets brise-vent de l'environnement paysager, pouvant être dus à différents types d'écrans (végé- taux ou topographiques). Dans notre cas, il s'agit principalement d'écrans végétaux, constitués de zones boisées plus ou moins importantes. L'IFP traduira tout d'abord un effet aérodynamique se manifestant par une réduction générale de la vitesse du vent, restant à moduler en fonction de la « perméabilité » du brise-vent associé (Guyot, 1997). Cet effet brise-vent est important et bien traduit par l'IFP, dans la mesure où la vitesse du vent mesurée est très bien corrélée négativement $(r=-0,96)$ à ce dernier pour les onze parcelles de l'étude (fig 8.a). La corrélation reste hautement significative $(r=-0,81)$ si l'on retire la parcelle extrême de clairière $1 \mathrm{ROC}$.

On notera que la variabilité de Rg est du même ordre que celle observée pour des régions qui présentent des pentes de 20 à $30 \%$ dont les orientations sont opposées (Lebon, 1993 ; Cellier, 1994). Là encore, ces variations peuvent être mises en relation directe avec l'IFP qui réduit significativement le rayonnement global comme le montre la fig 8 b. Le coefficient de corrélation de la relation présentée demeure hautement significatif même si l'on supprime la parcelle extrême IROC $(r=-0,70$ au lieu de $-0,78)$. Les masques Ouest et Est sont les plus influents. Le flux direct est diminué le soir pour 1 ROC et 2 ING, et le matin pour $1 \mathrm{CHA}$. Ce type de paysage à masques végétaux verticaux majoritaires fait apparaître les limites de l'utilisation des modèles d'estimation de l'énergie lumineuse reçue sur des plans inclinés et d'orientations variables, comme ceux de Varlet-Grancher (1975) ou Perrin de Brichambaut (1978). L'augmentation des températures derrière un brise-vent, citée par Guyot (1997), s'observe bien sur la courbe journalière du site IROC ( $f i g$ ). On note une température beaucoup plus élevée que la référence le matin et très inférieure le soir : le matin l'effet positif de "piège à rayonnement » et de brise-vent 

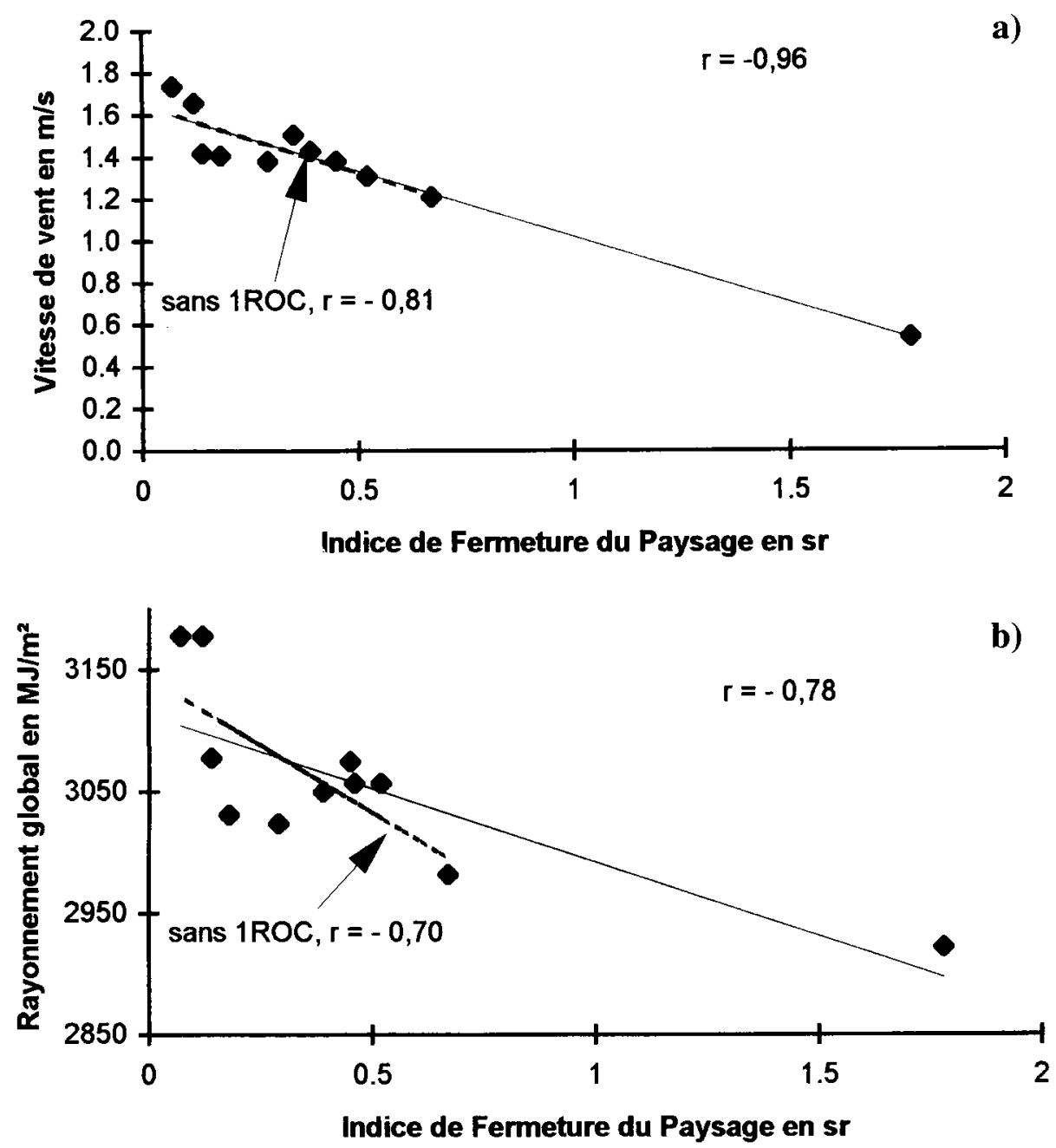

Fig 8.a. Relation entre l'indice de fermeture du paysage et les valeurs de vitesse du vent obtenues en 1998 sur onze sites. b. Relation entre l'indice de fermeture du paysage et les valeurs de rayonnement global obtenues en 1998 sur onze sites.

des bois proches domine, tandis que le soir, la forte diminution de l'éclairement direct, abaisse la température de l'air.

On peut tenter d'estimer l'effet résultant de l'IFP sur l'évapotranspiration potentielle climatique $(\mathrm{ETPc})$ grâce à la formule de Penman. A l'échelle journalière, cette formule peut s'écrire de la manière suivante :

$$
\mathrm{ETPc}=\mathrm{A} \cdot \mathrm{H}^{*} / \mathrm{L}+\mathrm{B} \cdot \mathrm{Ea}
$$

avec : $\mathrm{H}^{*}$ : exposition énergétique nette (valeur intégrée du rayonnement net à l'échelle de la journée); $L$ : chaleur latente de vaporisation de l'eau ; Ea : pouvoir évaporant de l'air ; A, B : constantes.

ETPc peut être considérée comme la somme d'un terme radiatif (A.H*/L) et d'un terme advectif (B.Ea). L'IFP modifiera donc $\mathrm{H}^{*}$, mais diminuera surtout $E a$, en raison de la réduction de la vitesse du vent. Sur les bases de la bibliographie, dans le vignoble étudié où la pluviométrie est infé- rieure à $600 \mathrm{~mm}$, les effets d'advection peuvent être estimés à $30 \%$ de ETPc. Finalement, on peut considérer que l'IFP pourrait réduire de $10 \%$ ETPc dans nos conditions. En effet, les calcul d'ETPc réalisés pour la période comprise entre le 2 juin et le 6 octobre 1988 donnent les résultats suivants : 417,444 et $446 \mathrm{~mm}$ respectivement pour $1 \mathrm{ROC}, 1 \mathrm{CHA}$ et $1 \mathrm{BOIS}$ qui sont les sites présentant les IFP les plus forts dans le même ordre (soit $1,78,0,67$ et $0,52 \mathrm{sr}$ ). À l'opposé, dans les parcelles IFON et 1TUR, l'ETPc a été de $489 \mathrm{~mm}$, pour des valeurs d'IFP de 0,18 et 0,12 . Le calcul montre effectivement une réduction d'ETPc comprise entre 9 et $15 \%$ lorsque IFP varie de 0,15 à $1,78 \mathrm{sr}$.

L'indice de fermeture du paysage semble un outil d'approche indirect, tout à fait intéressant pour traduire les effets climatiques de l'environnement paysager et particulièrement ceux liés aux écrans végétaux, comme nous venons de le pré- 
senter. Mais l'IFP peut aussi être utilisé pour quantifier les masques topographiques et leurs effets sur le rayonnement global, en vignobles de montagne (Dumas et al, 1997 ; Falcetti et al, 1993).

Dans la région étudiée, le relief peu accidenté présente moins de diversité que les sols ou l'environnement paysager. Mais, bien que la gamme d'altitude étudiée soit faible (42 à $108 \mathrm{~m}$ ), son effet connu sur la température s'exprime bien dans ce réseau. L'ordre de grandeur des variations de la température moyenne (environ $-0,5^{\circ} \mathrm{C}$ pour $60 \mathrm{~m}$ ) apparaît légèrement supérieur à ce que donne la bibliographie $(-0,65$ pour $100 \mathrm{~m})$. Des relations significatives entre altitude réelle et température moyenne de l'air, durant la période d'étude, ont pu être établies (tableau VIII ; fig 9). Les corrélations négatives entre altitude réelle et température sur la période de végétation sont améliorées $(-0,34$ à $-0,78)$ pour la température moyenne et $(-0,50$ à $-0,94)$ pour la température maximale, si l'on retire de l'analyse les parcelles du nord-est bourgueillois (+ froid) et 1ROC dont l'altitude forte est largement compensée par sa situation de clairière.

Les différences d'altitudes relatives permettent moins bien d'expliquer les écarts de température minimale, car les écoulements gravitaires d'air froid décrits par Godard (1949), sont limités par la douceur du relief. Toutefois, les parcelles 2 ING et 1POY situées en bas de versant et d'altitude relative négative, subissent cet effet. Les minimums moyens y sont respectivement inférieurs de $1{ }^{\circ} \mathrm{C}$ et $0,5^{\circ} \mathrm{C}$ par rapport aux parcelles situées immédiatement au-dessus (tableau VII) et on a aussi relevé des dégâts de gelée de printemps plus fréquentes et plus fortes dans ces terroir.
Tableau VIII. Principales corrélations entre facteurs physiques du paysage et température moyenne de l'air dans le réseau terroir du val de Loire.

\begin{tabular}{lccc}
\hline & $\begin{array}{c}\text { Alt réelle } \times \\
\text { Tmoy }\end{array}$ & $\begin{array}{c}\text { Alt réelle } \\
\text { Tmax }\end{array}$ & $\begin{array}{c}\text { \% sable } \\
\text { Tmoy }\end{array}$ \\
\hline 14 parcelles & $-0,34 \mathrm{~ns}$ & $-0,50 \mathrm{~ns}$ & $0,08 \mathrm{~ns}$ \\
11 parcelles & $-0,60 *$ & $-0,52 \mathrm{~ns}$ & $0,73 * *$ \\
10 parcelles & $-0,78 * *$ & $-0,94 * * *$ & $0,79 * *$ \\
\hline
\end{tabular}

14 parcelles = toutes parcelles étudiées, 11 parcelles $=$ les mêmes sans celles du nord-est du Bourgueillois (2ING, IING, IBOIS), 10 parcelles $=$ les 11 dernières moins 1 ROC

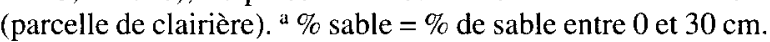

Nous n'avons pas noté d'influence positive de l'altitude sur les hauteurs des précipitations comme cela a pu être montré dans le bassin de Paris ou en Belgique pour des gammes d'altitude équivalente (Escourou, 1981). Les autres paramètres du relief comme la pente et l'exposition ne discriminent pas assez les UTB dans ce réseau pour modifier significativement l'insolation et l'interception du rayonnement solaire. En revanche, on note une relative augmentation de la vitesse du vent pour les parcelles 1ING et 1BOIS situées dans la partie supérieure de pentes douces orientées vers les vents dominants de S-O (fig 4). Cette observation rejoint celles de Guyot (1976) déjà citées dans l'introduction de ce mémoire.

On a pu aussi noter l'influence de la proximité d'une forêt importante sur certaine variables météorologiques. Ainsi, l'effet favorable des forêts sur la pluviosité (Doignon, 1951 ; Lee, 1978) semble jouer dans ce réseau. Pendant la période d'étude, la parcelle 2ING, située à moins de $100 \mathrm{~m}$ de la forêt bourgueuilloise a reçu $39 \mathrm{~mm}$

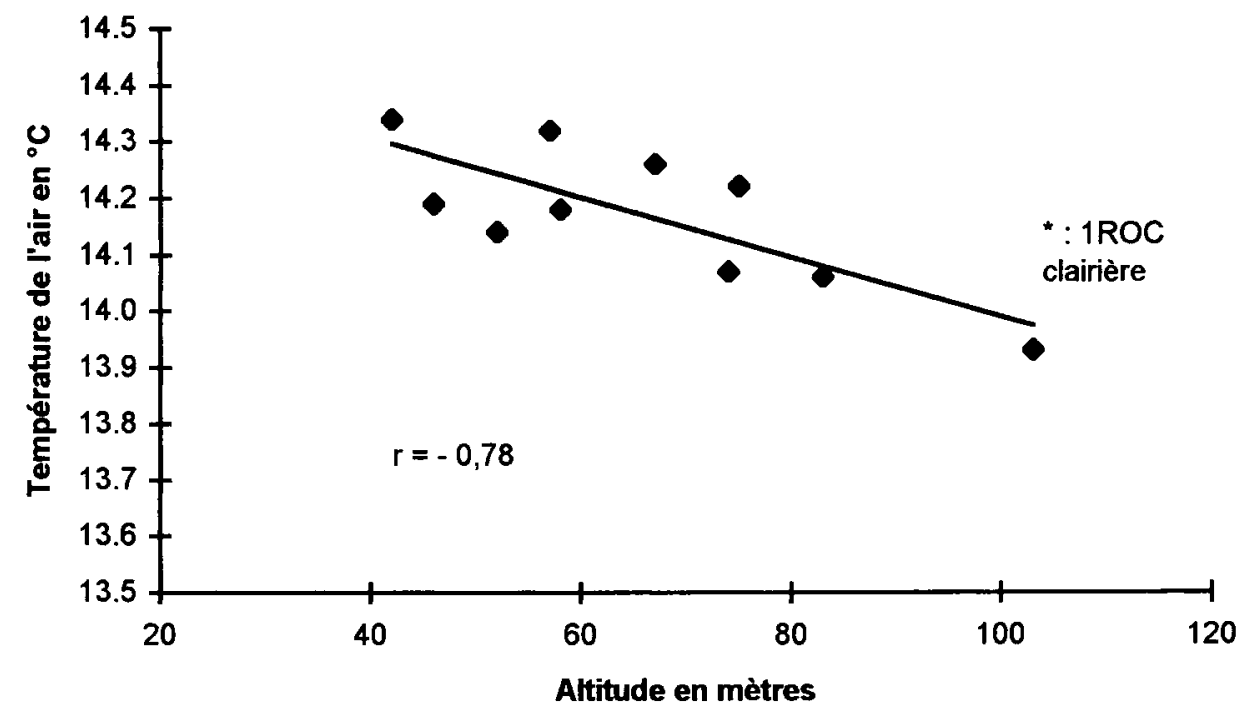

Fig 9. Variation de la température moyenne de l'air en fonction de l'altitude pour dix terroirs sur l'ensemble du cycle végétatif de la vigne en 1988. 
de plus que $1 \mathrm{ING}$ distante d'environ $1 \mathrm{~km}$ de cette forêt. À Chinon, c'est la parcelle 1ROC, en situation de quasi clairière qui a recueilli $13 \mathrm{~mm}$ de plus que 1VAU, située à quelques centaines de mètres de la lisière. Toutefois, pour cette situation, une amélioration du coefficient de captation du pluviomètre lié à la réduction de la vitesse du vent n'est pas à exclure. L'humidité de l'air est plus forte pour les parcelles situées en bordure de massifs forestiers (1ROC, 2ING, 1BOIS), en particulier pendant la phase végétative des arbres (tableaux V,VI ; fig 5). On peut émettre l'hypothèse que l'évapotranspiration de la forêt ainsi que son rôle sur la pluviosité, augmente l'humidité de l'air de ces situations où l'évacuation de la vapeur d'eau est diminuée par une réduction de la ventilation associée à de forts IFP.

\section{CONCLUSION}

Ce travail a mis en évidence l'existence de climats locaux (mésoclimats) associés à de petits territoires viticoles appelés UTB, caractérisés, identifiés et cartographiés à l'aide d'un méthode mise au point par Morlat (1989). Il a permis aussi de démontrer l'importance de l'environnement paysager du terroir, quantifié par l'indice de fermeture du paysage (IFP) proposé dans ce mémoire. Les variables climatiques les mieux reliées à cet indice sont la vitesse du vent dont dépend en partie l'évapotranspiration potentielle, l'interception du rayonnement solaire (très importante pour la photosynthèse et l'énergie disponible pour le réchauffement du sol et de l'air), et dans une moindre mesure sur l'humidité relative. L'altitude réelle s'est révélée être un critère opérationnel pour prédire la température moyenne et maximale dans la majorité des situations, bien que le relief soit peu accentué dans la région étudiée. En revanche, les relations entre altitude relative et températures minimales sont moins nettes, mais ce paramètre du relief mériterait une étude particulière orientée vers la définition des zones gélives. Le type de sol joue localement un rôle non négligeable sur la température et l'humidité de l'air. Les textures de surface sableuses influencent positivement les températures de l'air, tandis que les fortes capacités de rétention en eau des sols affectent positivement les teneurs en eau de l'air, par l'intermédiaire d'une évapotranspiration plus élevée du couvert de la vigne. La plupart de ces facteurs de variation, liés à la géopédologie locale, sont suffisamment accessibles pour être cartographiés à la même échelle que le sol $\left(1 / 25000^{\mathrm{e}}\right.$ par exemple) et permettre ainsi une véritable caractérisation intégrée des terroirs, représentative des différences de fonctionnement écophysiologique du système ter- roir-vigne. Néanmoins, dans un vignoble comme celui du val de Loire, des variables complémentaires comme les vents dominants, les isohyètes obtenus sur de longues périodes, et la situation des massifs forestiers sont aussi à retenir pour réaliser un zonage climatique.

Dans ce contexte, l'indice de fermeture du paysage, semble une variable suffisamment synthétique et représentative des modifications locales du climat régional, pour devenir un outil intéressant et opérationnel d'un zonage climatique de l'UTB. Toutefois, une étude plus fine, visant à préciser l'intérêt et les limites d'utilisation de cet indice, devrait être engagée pour le tester dans des situations géographiques différentes.

Dans une perspective de valorisation, il apparaît qu'il vaudrait mieux s'orienter vers des cartographies climatiques thématiques, car les différentes variables météorologiques ne sont pas toutes modifiées dans le même sens ni par les mêmes facteurs de l'environnement paysager. D'autre part, si des paramètres comme la température moyenne ou l'insolation peuvent se coupler aisément au potentiel de maturation du raisin, les températures minimales s'adressent plus aux risques de gel, et l'humidité relative à la gestion phytosanitaire de la vigne. Couplées à la dimension sol du terroir (aspect réserves hydriques par exemple), des approches spatialisées intégrant l'IFP (donc vitesse du vent et ETP), peuvent s'avérer très intéressantes pour une gestion agronomique des terroirs. Une collaboration étroite entre climatologues, bioclimatologistes, agronomes, épidémiologistes et oenologues est nécessaire afin d'optimiser cette valorisation.

Ces connaissances, associées à celles des autres facteurs naturels du terroir, devraient permettre dans l'avenir, de mieux définir la contribution de la composante climatique du terroir, à la qualité du vin produit et ainsi, de mieux intégrer le couple paysage $\mathrm{x}$ climat, dans les démarches de délimitation des zones d'appellation. Enfin, des approches concernant les fonctions de transfert du mésoclimat au microclimat sont à développer pour une meilleure connaissance de l'influence relative des facteurs naturels du climat d'une part, et des pratiques agroviticoles d'autre part.

Remerciements : Les auteurs tiennent à remercier Pierre Cellier qui les a fait bénéficier de ses conseils pour la mise au point de cet article.

\section{RÉFÉRENCES}

Bonnardot V (1997) Indices climatiques et estimation de la date des vendanges en Côte de Beaune (période 1975-1996). Revue des anologues 83, 35-38 
Branas J, Bernon G, Levadoux L (1946) Eléments de viticulture générale. Ensa, Montpellier, $400 \mathrm{p}$

Calo A, Costacurta A, Tomasi D, Becker N, Bourquin H.D, de Villiers F.S, Garcia de Lujan A, Huglin P, Jacquinet L, Lemaitre C (1992) La teneur en sucre du raisin : le déterminisme climatique. Rev Vitic Enol 3, 3-29

Cellier P (1994) Caractérisation microclimatique de la vigne dans le vignoble champenois : estimation et paramétrisation de la variabilité. Rapport d'avancement, non publié, Inra Bioclimatologie Grignon, France

Cellier P, Jacquet A, Bautrais P, Morlat R, Delanchy P (1996) Modélisation du régime thermique des sols de vignoble du val de Loire : relations avec des variables utilisables pour la caractérisation des terroirs. In : Actes du $1^{\text {er }}$ Colloque international sur les terroirs viticoles, Angers, France, 107-112

Chiapale JP (1975) A numerical model for estimating the modification of heat budget introduced by hedges. In : Proc Int Conf Heat and Mass Transfer in the Biosphere, Dubrovnik, Scripta Book Co, 457 466

Constantinescu G (1967) Méthodes et principes de détermination des aptitudes viticoles d'une région et du choix des cépages appropriés. Bull OIV 441, 1179-1205

Doignon P (1951) Le mésoclimat forestier de fontainebleau. Hygrométrie, nébulométrie, anémométrie, barométrie, conclusions. Centre régional de recherches naturalistes, Fontainebleau, $39 \mathrm{p}$

Dumas V, Lebon E, Morlat R (1997) Différenciations mésoclimatiques au sein du vignoble alsacien. J Int Sci Vigne Vin 31, 1-9

Durand R, Legros JP (1981) Cartographie automatique du rayonnement solaire en fonction du relief. agronomie 1, 31-39

Escourrou G (1981) Climat et environnement. Les facteurs locaux du climat. Masson, $182 \mathrm{p}$

Falcetti M, Bertamini M, Scienza A (1993) Criteri di definizione e di delimitazione delle aree viticole di montagna. Bollettino ISMA 3, 20-33

Godard M (1949) Microclimats et mésoclimats du point de vue agronomique, Ann Agron XIX, 578-604

Guyot G (1963) Les brise-vent. Modification du microclimat et amélioration de la production agricole. Ann Agron 14, 429-488

Guyot G (1997) Climatologie de l'environnement. Masson, Paris, 505 p

Guyot G, Bouchet RJ, Chiapale JP, Malet P, Seguin B, Verbrugghe $M$ (1976) Climat et aménagement en pays de bocage. Inra, Montfavet, rapport de fin de contrat d'étude, ministère de l'Agriculture et du Développement rural, $767 \mathrm{p}$

Guyot G, Bensalem B, Delécolle R (1987) Brise-vent et rideaux abris avec référence particulière aux zones sèches, Cahier de Conservation 15, FAO, Rome, $385 \mathrm{p}$
Huglin P (1978) Nouveau mode d'évaluation des possibilités héliothermiques d'un milieu viticole, $C R$ Acad Agric France 1117-1126

Huglin P (1986) Biologie et écologie de la vigne, Payot-Lausanne, technique et documentation, Paris, $371 \mathrm{p}$

Jacquet A, Morlat R (1996) Étude de la composante climatique du terroir viticole en val de Loire : relation avec les facteurs physiques du milieu. In : Actes $d u$ $1^{\text {er }}$ Colloque international sur les terroirs viticoles, Angers, France, 124-134

Jacquet A, Salette J, Cellier P, Morlat R, Fanet J (1995) Réflexion sur la notion d'échelle pour le terroir, conséquences pour le classement des sites viticoles. Revue des anologues 77S, 57-60

Lebon E (1993) De l'influence des facteurs pédo et méso-climatiques sur le comportement de la vigne et la maturation du raisin. Application à l'établissement de critères de zonage des potentialités qualitatives en vignoble septentrional (Alsace). Thèse de doctorat en géologie, université de Dijon, $165 \mathrm{p}$

Lee R (1978) Forest Microclimatology. Colombia Univ Press, New York, 276 p

Morlat R (1989) Le terroir viticole : contribution à l'étude de sa caractérisation et de son influence sur les vins. Applications aux vignobles rouges de moyenne vallée de la Loire. Thèse d'État, université Bordeaux-II, $289 \mathrm{p}+$ annexes

Morlat R (1996) Éléments importants d'une méthodologie de caractérisation des facteurs naturels du terroir, en relation avec la réponse de la vigne à travers le vin. In : : Actes du $I^{\text {er }}$ Colloque international sur les terroirs viticoles, Angers, France, 17-31

Morlat R, Jacquet A (1993) The soils effects on the grapevine root system in several vineyards of the Loire Valley (France). Vitis 32, 35-42

Perrin De Brichambaut C (1978) Estimation de l'énergie solaire disponible au sol. La Météorologie VI (15), 5-45

Restoin MA (1993) Typologie du pédoclimat thermique de divers terroirs viticoles en val de Loire : Relations avec le comportement de la vigne. Mémoire fin d'études, Enita, Bordeaux, $45 \mathrm{p}$ et annexes

Ribéreau-Gayon J, Peynaud E (1971) Traité d'ampélologie, tome 1, Dunod, $725 \mathrm{p}$

Ribéreau-Gayon J et al (1975). Sciences et techniques de la vigne et du vin, tome 2, Dunod

Riou C et al (1994) Le déterminisme climatique de la maturation du raisin. Application au zonage de la teneur en sucre dans l'Union européenne. EUR 15863-FR/EN, Office des publications officielles de l'Union européenne, Luxembourg, 322p + cartes

Riou C, Morlat R, Asselin C (1995) Une approche intégrée des terroirs viticoles. Discussions sur les critères de caractérisations accessibles. Bull de l'OIV $68,767 / 768,94-106$ 
Samie C (1983) Caractérisation climatique locale : micro/mésoclimats. Bull OEPP 13, 15-20

Varlet Grancher C (1975) Variation et estimation de l'énergie d'origine solaire reçue sur des plans d'inclinaison et d'azimut variables. Ann Agron 26, 245-264

Verbrugghe M, Guyot G, Hanocq JF, Ripoche D (1991) Influence de différents types de sol de la basse val- lée du Rhône sur les températures de surface de raisins et de feuilles de Vitis vinifera. Revue Française d'anologie 128, cahier scientifique.

de Vries DA (1963) Thermal properties of soils. In : Physics of Plant Environnement (Van Wijk, ed.), North-Holland, Amsterdam, 210-235

Winkler A.J (1962) General Viticulture. Univ of California Press 